\title{
Monoacylglycerol O-acyltransferase 1 (MGAT1) localizes to the ER and lipid droplets promoting triacylglycerol synthesis
}

\author{
Yoo Jeong Lee ${ }^{1}$ E Jae-woo Kim, Ka, * $^{2,4}$ \\ ${ }^{1}$ Division of Metabolic Disease, Center for Biomedical Sciences, National Institutes of Health, Cheongju 28159, ${ }^{2}$ Department of \\ Biochemistry and Molecular Biology, Integrated Genomic Research Center for Metabolic Regulation, Institute of Genetic Science, Yonsei \\ University College of Medicine, ${ }^{3}$ Brain Korea 21 PLUS Project for Medical Science, Yonsei University, ${ }^{4}$ Severance Biomedical Science \\ Institute, Yonsei University College of Medicine, Seoul 03722, Korea
}

\begin{abstract}
Monoacylglycerol acyltransferase 1 (MGAT) is a microsomal enzyme that catalyzes the synthesis of diacylglycerol (DAG) and triacylglycerol (TAG). However, the subcellular localization and catalytic function domain of this enzyme is poorly understood. In this report, we identified that murine MGAT1 localizes to the endoplasmic reticulum (ER) under normal conditions, whereas MGAT1 co-localize to the lipid droplets (LD) under conditions of enriching fatty acids, contributing to TAG synthesis and LD expansion. For the enzyme activity, both the $\mathrm{N}$-terminal transmembrane domain and catalytic HPHG motif are required. We also show that the transmembrane domain of MGAT1 consists of two hydrophobic regions in the $\mathrm{N}$-terminus, and the consensus sequence FLXLXXXn, a putative neutral lipid-binding domain, exists in the first transmembrane domain. Finally, MGAT1 interacts with DGAT2, which serves to synergistically increase the TAG biosynthesis and LD expansion, leading to enhancement of lipid accumulation in the liver and fat. [BMB Reports 2017; 50(7): 367-372]
\end{abstract}

\section{INTRODUCTION}

Nonalcoholic fatty liver disease (NAFLD) is commonly found in patients with metabolic syndrome without extensive consumption of alcohol (1), encompassing liver lesions ranging from steatosis to nonalcoholic steatohepatitis (NASH), cirrhosis and even liver cancer (2). It was reported that about $75 \%$ people who have hepatic steatosis are obese (3). Obesity with insulin resistance causes an increased lipolysis in adipose

${ }^{*}$ Corresponding author. Tel: +82-2-2228-0837; Fax: +82-2-3125041; E-mail: japol13@yuhs.ac

https://doi.org/10.5483/BMBRep.2017.50.7.036

Received 6 March 2017, Revised 16 March 2017, Accepted 22 March 2017

Keywords: DGAT2, Hepatic steatosis, Lipid droplet, MGAT1, Triacylglycerol synthesis tissue, thus resulting in an increased delivery of free fatty acids (FFAs) to the liver, contributing to excessive hepatic lipid accumulation (4). In turn, this impairs the hepatic functions, leading to a spectrum of disorders characterized by liver steatosis.

Mammalian cells synthesize triacylglycerol (TAG) via two convergent pathways. The classic pathway, called glycerol3-phosphate pathway, starts first with glycerol-3-phosphate acyltransferase (GPAT) which catalyzes the acylation of glycerol-3-phosphate forming lysophosphatidic acid. Then 1-acylglycerol-3-phosphate acyltransferase (AGPAT) and lipin act to further acylate and dephosphorylate the lysophosphatidic acid, respectively, to produce diacylglycerol (DAG). On the other hand, the monoacylglycerol acyltransferase (MGAT) promotes alternative pathway to synthesize TAG. This enzyme directly catalyze the acylation of monoacylglycerol (MAG) to produce DAG. Finally, DAG is converted to TAG by DGAT1 or DGAT2, which is common in both pathways $(5,6)$.

Since the liver is not the primary fat storage depot, the steady state concentration of hepatic triglycerides is low under physiological conditions. However, with overeating and lack of exercise, the liver stores the excess energy as TAG. NAFLD associated with obesity is contributed largely by greater fatty acid release from the adipose tissue. Elevated levels of FFA in plasma increase the delivery of FFA to the liver, resulting in excessive hepatic TAG accumulation. Therefore, in the obesity-related hepatic steatosis, FFAs from adipose tissue and dietary fat directly enter the TAG synthesis pathway, probably through the GPAT pathway, as well as alternative pathways using increased MGAT1 enzyme, resulting in rapid TAG incorporation (7). In the last decade, a family of three mammalian genes encoding enzymes with MGAT activity have been identified (8-10). MGAT1 was originally identified in mice as a microsomal enzyme that catalyzes the synthesis of DAG and TAG (8). MGAT2 is predominantly expressed in the small intestine, and plays a role in dietary fat absorption (11). MGAT3, which shares a higher sequence homology with DGAT2, is found only in higher mammals and humans, but not in rodents (12-14). All three MGAT family genes are localized in the endoplasmic reticulum (ER), but differ in tissue 
expression patterns and in catalytic properties (15). Recently, we reported that MGAT1 expression is upregulated in hepatic steatosis, and the adenovirus-mediated shRNA for MGAT1 critically reduces lipid accumulation in the liver $(16,17)$. However, little is known how the MGAT1 enzyme acts in the cell, including at its subcellular localization. Because MGAT1 is considered as an effective target for reducing hepatic steatosis and obesity $(16,17)$, it is now important to uncover the characteristics of the enzyme in lipid accumulation.

In this report, we demonstrate that MGAT1 is an integral ER membrane protein, and consists of two transmembrane domains. In addition to existing at the ER, MGAT1 is also localized in lipid droplets. We further determined that LD-localized MGAT1 may contribute to the growth of TG-containing lipid droplets, through the HPHG residue which is associated with catalytic activity of the enzyme. The heterodimer formed by MGAT1 with DGAT2 synergistically increases the TG biosynthesis and LD expansion, suggesting that MGAT1-DGAT2 coordination plays an important role in the progression of hepatic steatosis.

\section{RESULTS}

\section{MGAT1 is an integral ER membrane protein and promotes TG synthesis}

Synthesis of TAG is through a distinct MGAT-dependent pathway: MAG is converted to DAG by MGAT enzymes, and DAG is converted to TAG by DGAT (Fig. 1A). Our previous studies suggest that MGAT1 has an important role in fatty liver formation, and a new target gene of PPAR $\gamma$ (17-19). We analyzed the expression patterns of MGAT1 in high-fat-fed liver and 3T3-L1 adipocytes, the two main tissues of lipid accumulation. As shown in Fig. 1B, MGAT1 is highly upregulated in fatty liver, as previously reported (17). Moreover, MGAT1 expression dramatically increased during adipogenesis, and escalated approximately 70 -fold at 8 days. To confirm the role of MGAT1 in lipid accumulation, we used confocal immunofluorescence microscopy in COS-7 cells. Under oleate loading, the overexpression of MGAT1 led to the formation of lipid droplets (LDs) (Fig. 1C). Moreover, when MGAT1 was co-expressed with DGAT2, the LDs were significantly larger than those of cells expressing MGAT1 alone.

Mouse MGAT1 is a 335-aa polypeptide, and the sequence of MGAT1 predicts one or two transmembrane domains, which is similar to DGAT2 (8). To clarify the transmembrane domain of MGAT1, we expressed different Flag-tagged deletion mutants of MGAT1 in HEK293T cells, followed by membrane fractionation (Fig. 1D). The mutant FL- $\Delta 1-160$, with both transmembrane regions deleted, displayed relocation in the cytosol. In contrast, like FL-MGAT1, mutant FL- $\Delta 161-335$, which contained the $\mathrm{N}$-terminus and the two transmembrane domains, exists in the membrane fraction. Consistently, MGAT1 displayed a typical ER staining pattern, whereas when transmembrane domains were deleted from MGAT1
(FL- $\Delta 1-160)$, the MGAT1 displayed a diffuse cytoplasmic staining pattern (Fig. 1D), thereby demonstrating that the transmembrane domain of MGAT1 consists of two hydrophobic regions at the $\mathrm{N}$-terminus.

We next examined whether the $\mathrm{N}$ and $\mathrm{C}$ termini of MGAT1 are localized in the cytoplasm or the ER lumen. Protease protection studies employing proteinase $\mathrm{K}$ treatment demonstrated that the Flag tags fused to either terminus of MGAT1 were digested by proteinase $K$, while the ER luminal protein GRP78 remained undigested by protease (20), indicating that the $\mathrm{N}$ and the $\mathrm{C}$ termini of MGAT1 are localized in the cytoplasm (Fig. 1E). These results suggest that MGAT1 has a number of membrane-inserted helices, and the residues 1-160 of MGAT1 include the transmembrane domain containing the ER targeting signal.

Highly conserved HPHG is important for MGAT1 activity Next, we explored the enzymatic activity of MGAT1. Intriguingly, enzymatic activity was absent when the

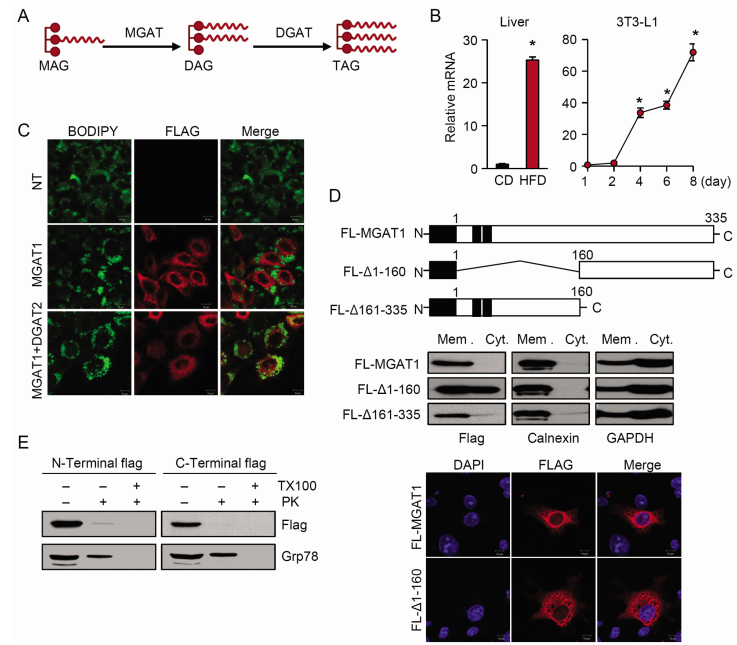

Fig. 1. MGAT1 is an integral ER membrane protein. (A) The MAG pathway involved in the synthesis of TAG. (B) Real-time PCR analysis of MGAT1 mRNA levels in the liver from chow-diet (CD) and high fat diet (HFD)-fed mice, and levels during adipogenesis of 3T3-L1 cells. (C) Immunofluorescent staining showing expression of FL-MGAT1 (red) on forming LDs (BODIPY, green) in oleate-loaded COS-7 cells. (D) Schematic representation of the MGAT1 structure using MGAT1 deletion mutant protein. HEK293T cells were transiently transfected with FL-MGAT1 and the indicated MGAT1 mutants. Total cellular membranes and cytosol were isolated and immunoblotted with anti-FLAG, anti-calnexin (ER marker), and anti-GAPDH (cytosolic marker) antibodies. COS-7 cells expressing FL-MGAT1 or FL- 1 1-160 were stained with anti-FLAG. Scale bars, $10 \mu \mathrm{m}$. (E) Protease protection. Proteinase K (PK) was added to intact microsomes with or without Triton X-100. A representative experiment out of three with similar outcome is shown. ${ }^{* P}<0.05$. Data represent the mean \pm SEM. 
transmembrane domains were deleted (Fig. 2A) Moreover, the mutant FL- $\Delta 101-335$ also lost its enzyme activity (Fig. 2A), suggesting that this domain has a catalytic motif. MGAT1 has homology with DGAT2, and also contains a highly conserved HPHG, a characteristic motif of the DGAT2 family (21). Alignment of the MGAT family members shows that the motif HPHG is conserved in sequences from animals and fungi (Fig. 2B). Four mutants of HPHG (109-112 aa of murine MGAT1) were generated by conservative substitution: $\mathrm{H} 109 \mathrm{~A}, \mathrm{P} 110 \mathrm{G}$, $\mathrm{H} 111 \mathrm{~A}$, and a triple mutant in which $\mathrm{HPH}$ was changed to AGA. Their expressions were confirmed by immunoblotting with anti-FLAG antibodies. As a result, all four mutants caused an approximately $60-70 \%$ decrease in enzymatic activity, compared to control FL-MGAT1 (Fig. 2C). We also performed TAG synthesis by BODIPY and immunofluorescent staining in COS-7 cells. In oleate-treated cell, the wild-type MGAT1 led to the formation of lipid droplets (LDs), whereas in cells expressing mutants, there were no LDs (Fig. 2D), suggesting that the conserved HPHG residue is associated with catalytic activity of enzyme, and the transmembrane domain is also required for the enzyme activity.

\section{MGAT1 is associated with lipid droplets}

Neutral lipids are synthesized by enzymes which mainly
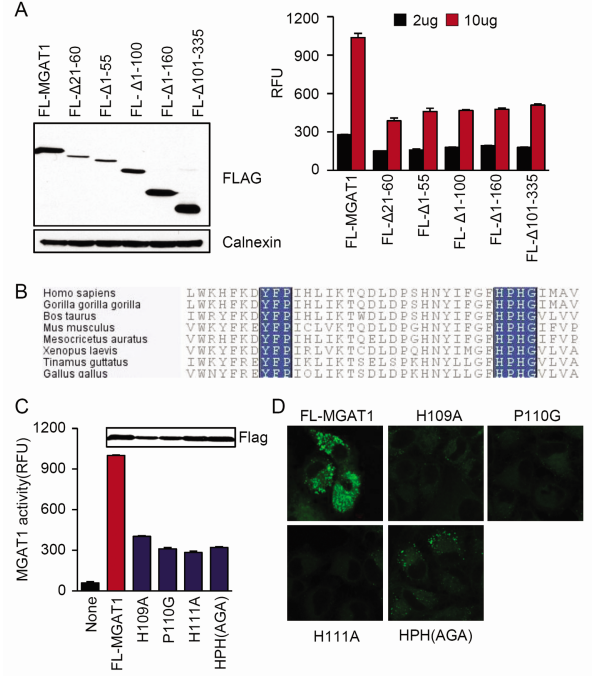

Fig. 2. Deletion mutants of MGAT1 have no effect on enzyme activity. (A) Immunoblot analysis of total membrane fraction isolated from COS-7 cells transfected with the indicated deletion mutants (left). The enzymatic activity determined in membrane fraction by measuring the fluorescence signal (right). (B) Alignment of partial sequence of MGAT1 members. A highly conserved HPHG sequence is indicated by a blue box. (C) The enzymatic activity determined in membrane fraction by measuring the fluorescence signal. (D) Immunofluorescence microscopy of lipid droplets in COS-7 cells treated with oleate. These cells were labeled with the neutral lipid stain BODIPY 495/503 (green). One representative experiment of three is shown. localize to the ER; likewise, newly formed lipid droplets also originate from the ER (22). Consequently, LD growth occurs by the local synthesis of TAG at the surface of LD, indicating a demand of enzymes necessary for TAG synthesis (23). To explore whether MGAT1 is present on LD surface, cellular membranes and the floating fat layer containing lipid droplets were separately isolated from fully differentiated-adipocytes and HeLa cells expressing MGAT1. As expected, MGAT1 localizes to the lipid droplets (Fig. 3A), as a similar pattern with previous reports that DGAT2 and GPAT4 localize to LDs $(24,25)$. Western blot analysis revealed that FL-MGAT1 is presented in the fat layer as well as in the membrane fraction, whereas constructs lacking the $\mathrm{N}$-terminus or $\mathrm{C}$-terminus could not be detected in the fat layer (Fig. 3B). Interestingly, FL- $\Delta$ 1-160 lacked both transmembrane domains and the conserved HPHG residue; in contrast, FL- $\Delta 161-335$ possessed both, but lacked the C-terminus. Consequentially, these results propose the possibility that both the HPHG residue and the C-terminal region are essential for the LD localization of MGAT1. To confirm that MGAT1 localized to lipid droplets, we co-stained MGAT1-transfected cells with the neutral lipids dye, BODIPY 493/503. As shown in Fig. 3C, MGAT1 was co-localized with the lipid droplets, indicating that LD-localized MGAT1 may contribute to the growth of TAG-containing lipid droplets.

\section{MGAT1 interacts with DGAT2}

Recent studies have shown that DGAT1 and DGAT2 form

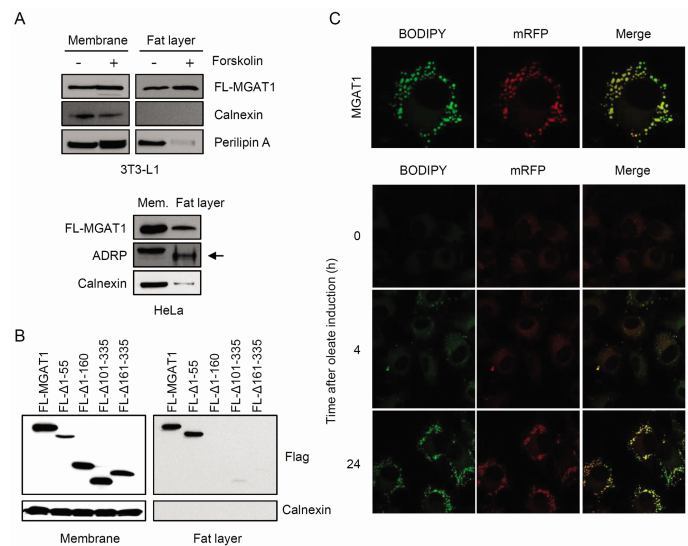

Fig. 3. MGAT1 is present in the lipid droplet. (A) Total cellular membranes and fat layers were isolated from fully differentiated 3T3-L1 adipocytes and HeLa cells transfected with Flag-MGAT1. Western blots of equal protein amounts against FLAG, Calnexin, Perilipin A, and ADRP are shown. (B) Immunoblot for indicated proteins in total cellular membranes or fat layers from HEK293T cells transfected with the indicated deletion mutants. (C) COS-7 cells transfected with mRFP-tagged MGAT1 were fixed with paraformaldehyde and were stained for neutral lipid with BODIPY 495/503 (green). A representative experiment out of three with similar outcome is shown. 
homodimers and homotetramers (26, 27), and MGAT2 can exist as both a homodimer and homotetramer $(28,29)$. Since MGAT1 shares sequence homology with MGAT2, and the $\mathrm{N}$-terminus of MGAT1 contains transmembrane domains, we investigated whether MGAT1 can form a homodimer. Cell lysates containing Myc-MGAT1 and various FLAG-MGAT1 deletion mutants were immunoprecipitated with anti-Myc, after which they were immunoblotted with anti-FLAG. Deletion of transmembrane domains $(\Delta 1-160)$ abolished the interaction between two MGAT1 monomers, but the MGAT1 mutant containing only the $\mathrm{N}$-terminus and transmembrane domains interacted with each other (Fig. 4A). It has been shown that MGAT and DGAT catalyze the two consecutive steps in TAG synthesis, and as shown in Fig. 1B, the co-expression of MGAT1 and DGAT2 promotes TG synthesis. Thus, we next examined whether MGAT1 heterodimerizes with DGAT2. As verified by co-immunoprecipitation, MGAT1 interacts with DGAT2 (Fig. 4B). However, the N-terminal deletion mutant $(\Delta 1-160)$ of MGAT1 was a blunted heterodimer with DGAT2, due to removal of the transmembrane domains, while DGAT2 interacted strongly with C-terminal deletion mutants of MGAT1 ( $\Delta 161-335)$. Taken together, these results suggested that MGAT1 localizes to both the ER and LDs, and the transmembrane domains are important for the enzyme activity as well as for the interaction with each other.

\section{DISCUSSION}

Since LD is now considered as a cellular organelle, regulation
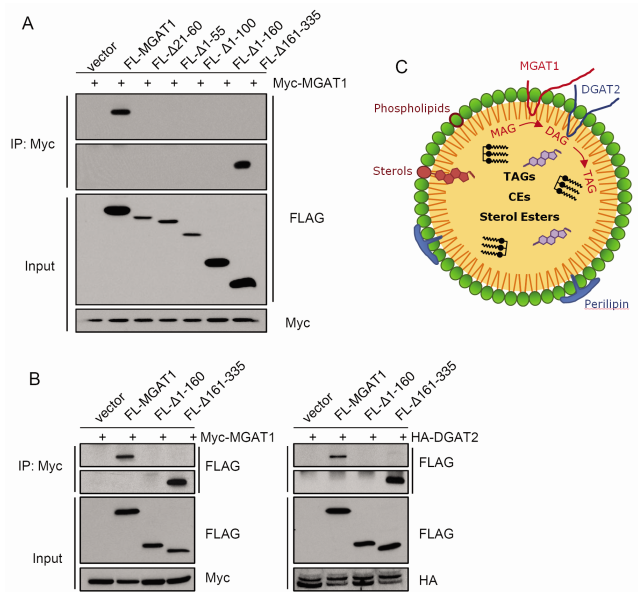

Fig. 4. MGAT1 interacts with DGAT2. Co-immunoprecipitation experiments detecting (A) the interaction between myc-MGAT1 and FL-MGAT1, and (B) the interaction between HA-DGAT2 and FL-MGAT1 in HEK293T cells transfected with the indicated deletion mutants. The precipitated proteins were subjected to western blot analysis with FLAG antibody. A representative experiment out of three with similar outcome is shown. (C) Model of MGAT1 anchored in lipid droplet. of the formation, growth, and degradation has now attracted considerable attention. Because it is believed that fatty acids are directly transported and incorporated into LDs after being separated from ER by budding, the LD should contain an independent machinery for lipid accumulation, including the TAG synthesis enzymes. Our data revealed that TAG synthesis is supported by luminally oriented acyltransferases, such as MGAT1 and DGAT2. (Fig. 4C). We previously demonstrated that MGAT1 expression is induced by PPAR $\gamma$ which is aberrantly overexpressed in steatotic liver, and liver-specific disruption of MGAT1 dramatically ameliorates hepatic steatosis associated with diet-induced obesity $(16,17)$. In addition, according to recent researches, inhibiting the MGAT1 activity improves hepatic metabolic abnormalities and insulin signaling $(30,31)$. In accordance with this, we observed that the intracellular contents of lipid droplets were increased by MGAT1 in the presence of FFA, but LDs barely generated in the cells without fatty acid treatment. Thus, these results indicated that under FFA loading, MGAT1 facilitates TAG synthesis and LD expansion.

Deletion of the hydrophobic transmembrane domain leads to diffuse cytosolic staining. Evidence suggests that this region might be critical for localization for MGAT1 to ER. Our data indicate that MGAT1 harbors maybe two or more hydrophobic domains, with both the $\mathrm{N}$-terminal and the $\mathrm{C}$-terminal portions extending toward the cytosol. Notably, MGAT1 contains the consensus sequence FLXLXXXn (where $\mathrm{n}$ is a nonpolar residue and $X$ is any amino acid except proline) that is conserved for a putative neutral lipid-binding domain in the transmembrane domain (amino acids ${ }^{28} \mathrm{FLLLVQV}^{34}$ ) (32). This sequence is most highly conserved in the vertebrate DGAT2 and MGAT orthologues (23) and other proteins that either interact or metabolize neutral lipids (32). Moreover, MGAT1 contains the catalytic HPHG motif (109-112 of murine MGAT1), and mutations of amino acids within this sequence significantly reduce the MGAT1 catalytic function. Intriguingly, we detected high enzyme activity only in full-length FL-MGAT1, while other deletion mutants failed to catalyze the synthesis of diacylglycerol. We speculate the possibility that neutral lipid-binding domain and catalytic HPHG motifs, as well as C-terminus region and other elements, are required for MGAT1 activity. Further experiments are required to determine the function of these active site domains.

DGAT1 has been shown to form a homodimer or homotetramer (27), and heterodimerize with MGAT2 to mediate dietary fat absorption (28). Similarly, DGAT2 is part of a multimeric complex (16), and interacts with other TG synthesis enzymes, such as fatty acid transport protein 1 (FATP1), stearoyl CoA desaturase 1 (SCD1), and MGAT2, to facilitate TG synthesis, resulting in an expansion of lipid droplets. We found that MGAT1 also forms homodimer through the N-terminal part, possibly homotetramer like MGAT2 or DGAT, and also interacts with DGAT2 to promote TAG synthesis. In addition, although MGAT1 is thought to be 
mainly regulated by gene expression, it will be interesting to examine whether a certain stimulus (i.e. fatty acid loading) induces the translocation of MGAT1 to LDs.

In conclusion, our studies indicate that MGAT1 is localized to the ER and lipid droplets, where it catalyzes the formation of DAG from MAG and fatty acyl-CoA. Transmembrane domain of MGAT1 consists of hydrophobic regions in the $\mathrm{N}$-terminus, and both $\mathrm{N}$ and $\mathrm{C}$ termini are localized to the cytosol. Importantly, MGAT1 interacts with DGAT2, which serves synergistic increase in TG biosynthesis and LD expansion, resulting in enhanced lipid accumulation in the liver.

\section{MATERIALS AND METHODS}

\section{Cell culture and transfection}

COS-7 and HEK293T cells were maintained in Dulbecco's modified Eagle's medium supplemented with 10\% (vol/vol) fetal bovine serum (Invitrogen). Transient transfection was performed with Lipofectamine LTX, according to the manufacturer's protocol (Invitrogen). Cells were harvested and used for experiments $48 \mathrm{~h}$ after transfection.

\section{Construction of MGAT1 plasmids}

N-terminal FLAG tagged murine MGAT1 (FL-MGAT1) was used as a template for all mutagenesis reactions. Site-directed mutagenesis was carried out with Pfu Turbo DNA Polymerase, according to the manufacturer's protocol (Agilent), and the presence of the desired mutations was confirmed by sequencing.

\section{Cellular fractionation}

Cells were harvested, washed with ice-cold PBS, and re-suspended in STE buffer ( $250 \mathrm{mM}$ sucrose, $50 \mathrm{mM}$ Tris-HCl, $\mathrm{pH} 7.4,1 \mathrm{mM}$ EDTA, and protease inhibitor). Cells were homogenized by 10 passages through a 27-gauge syringe needle, and lysates were cleared by 10 min centrifugation at $600 \times \mathrm{g}$, and $15 \mathrm{~min}$ at $12,000 \times \mathrm{g}$, to pellet the crude mitochondria. The supernatant was then centrifuged at $100,000 \times \mathrm{g}$ for $1 \mathrm{~h}$ at $4^{\circ} \mathrm{C}$ to pellet microsomes. The supernatant was used as the cytosolic fraction.

\section{Immunofluorescence}

Cells were washed with PBS, fixed in $4 \%$ paraformaldehyde for 10 min, washed with PBS and blocked with $3 \%$ BSA in PBS for $1 \mathrm{~h}$. Cells were incubated in primary antibody for 2 hour at room temperature (RT), followed by incubation with appropriate secondary antibody for 1 hour at RT. Cells were stained for lipid droplets with BODIPY493/503 $(1 \mu \mathrm{g} / \mathrm{ml}$, Molecular Probes) and for nuclei with Hoechst 33342 (Molecular Probes). Slides were mounted. Confocal scanning was performed on a LSM700 scanning microscope (Carl Zeiss).

\section{Immunoprecipitation}

Whole cell protein extracts were obtained from 293T cells using a lysis buffer ( $1 \% \mathrm{NP}-40,25 \mathrm{mM}$ HEPES, $150 \mathrm{mM} \mathrm{NaCl}$,
$2 \mathrm{mM}$ EGTA) containing protease and phosphatase inhibitor cocktail. Extracts were incubated overnight with $2.5 \mu \mathrm{g}$ of anti-myc antibody (Santa Cruz Biotechnology) in the presence of Protein G beads. The resulting complexes were washed, denatured and eluted. The IP samples and whole cell extracts were analyzed by immunoblotting performed with anti-Flag antibody (Sigma).

\section{MGAT activity assays}

In this assay, MGAT1 activity was measured as described previously (33). Briefly, the assay was carried out in a total volume of $150 \mu \mathrm{l}$ under the following conditions: $100 \mathrm{mM}$ Tris- $\mathrm{HCl}, \mathrm{pH}$ 7.4, $200 \mu \mathrm{M}$ 2-monoacylglycerol, $100 \mathrm{uM}$ oleoyl-CoA, and $10 \mu \mathrm{g}$ of microsomal proteins. The reaction was initiated by addition of MGAT1 microsomes, and carried out for $30 \mathrm{~min}$ at room temperature. Then, $50 \mu \mathrm{l}$ of 7-Diethylamino-3-(4-maleimidophenyl)-4-methylcoumarin (CPM) at $500 \mu \mathrm{M}$ concentration, was added to the reaction; the plate was incubated at room temperature for another 30 min. A standard curve of $\mathrm{CoASH}$ (ranging from 0 to $100 \mu \mathrm{M}$ of $\mathrm{CoASH}$ ) was generated together with each assay, followed by detection of fluorescent signal by a Thermo Scientific varioskan flash (Ex. 355 nm, Em. 460 nm).

\section{Statistical analysis}

All results are expressed as mean \pm SEM. Statistical comparisons of groups were made using an unpaired Student's t test.

\section{ACKNOWLEDGEMENTS}

This work was supported by the National Research Foundation of Korea (NRF) Grants 2011-0030086, 2015R1A2A2A01004345, and 2015R1A2A1A15055583, funded by the Korean government, Ministry of Science, ICT and Future Planning (MSIP).

\section{CONFLICTS OF INTEREST}

The authors have no conflicting financial interests.

\section{REFERENCES}

1. Marchesini G, Brizi M, Bianchi G et al (2001) Nonalcoholic fatty liver disease: a feature of the metabolic syndrome. Diabetes 50, 1844-1850

2. Angulo $P(2002)$ Nonalcoholic fatty liver disease. N Engl J Med 346, 1221-1231

3. Hamaguchi M, Kojima T, Takeda N et al (2005) The metabolic syndrome as a predictor of nonalcoholic fatty liver disease. Ann Intern Med 143, 722-728

4. Brunt EM (2010) Pathology of nonalcoholic fatty liver disease. Nat Rev Gastroenterol Hepatol 7, 195-203

5. Phan CT and Tso P (2001) Intestinal lipid absorption and transport. Front Biosci 6, D299-319

6. Lee YJ, Yu JH, Kim WH and Kim JW (2014) New mechanisms contributing to hepatic steatosis: glucose, 
insulin, and lipid signaling. Anim Cells Syst 18, 77-82

7. Shi Y and Cheng D (2009) Beyond triglyceride synthesis: the dynamic functional roles of MGAT and DGAT enzymes in energy metabolism. Am J Physiol Endocrinol Metab 297, E10-8

8. Yen CL, Stone SJ, Cases S, Zhou P and Farese RV Jr (2002) Identification of a gene encoding MGAT1, a monoacylglycerol acyltransferase. Proc Natl Acad Sci U S A 99, 8512-8517

9. Cheng D, Nelson TC, Chen J et al (2003) Identification of acyl coenzyme A:monoacylglycerol acyltransferase 3, an intestinal specific enzyme implicated in dietary fat absorption. J Biol Chem 278, 13611-13614

10. Yen CL and Farese RV Jr (2003) MGAT2, a monoacylglycerol acyltransferase expressed in the small intestine. J Biol Chem 278, 18532-18537

11. Cao J, Hawkins E, Brozinick J et al (2004) A predominant role of acyl-CoA:monoacylglycerol acyltransferase-2 in dietary fat absorption implicated by tissue distribution, subcellular localization, and up-regulation by high fat diet. J Biol Chem 279, 18878-18886

12. Yue YG, Chen YQ, Zhang $Y$ et al (2011) The acyl coenzymeA:monoacylglycerol acyltransferase 3 (MGAT3) gene is a pseudogene in mice but encodes a functional enzyme in rats. Lipids 46, 513-520

13. Cao J, Cheng $L$ and Shi $Y$ (2007) Catalytic properties of MGAT3, a putative triacylgycerol synthase. J Lipid Res 48, 583-591

14. Holmes RS (2010) Comparative genomics and proteomics of vertebrate diacylglycerol acyltransferase (DGAT), acyl CoA wax alcohol acyltransferase (AWAT) and monoacylglycerol acyltransferase (MGAT). Comp Biochem Physiol Part D Genomics Proteomics 5, 45-54

15. Lockwood JF, Cao J, Burn P and Shi Y (2003) Human intestinal monoacylglycerol acyltransferase: differential features in tissue expression and activity. Am J Physiol Endocrinol Metab 285, E927-937

16. Hayashi Y, Suemitsu E, Kajimoto K et al (2014) Hepatic Monoacylglycerol O- acyltransferase 1 as a Promising Therapeutic Target for Steatosis, Obesity, and Type 2 Diabetes. Mol Ther Nucleic Acids 3, e154

17. Lee YJ, Ko EH, Kim JE et al (2012) Nuclear receptor PPARgamma-regulated monoacylglycerol O-acyltransferase 1 (MGAT1) expression is responsible for the lipid accumulation in diet-induced hepatic steatosis. Proc Natl Acad Sci U S A 109, 13656-13661

18. Hall AM, Kou K, Chen Z et al (2012) Evidence for regulated monoacylglycerol acyltransferase expression and activity in human liver. J Lipid Res 53, 990-999

19. Yu JH, Lee YJ, Kim HJ et al (2015) Monoacylglycerol O-acyltransferase 1 is regulated by peroxisome proliferator-activated receptor gamma in human hepatocytes and increases lipid accumulation. Biochem Biophys Res Commun 460, 715-720

20. Reddy RK, Mao C, Baumeister P, Austin RC, Kaufman RJ and Lee AS (2003) Endoplasmic reticulum chaperone protein GRP78 protects cells from apoptosis induced by topoisomerase inhibitors: role of ATP binding site in suppression of caspase-7 activation. J Biol Chem 278, 20915-20924

21. Stone SJ, Levin MC and Farese RV Jr (2006) Membrane topology and identification of key functional amino acid residues of murine acyl-CoA:diacylglycerol acyltransferase-2. J Biol Chem 281, 40273-40282

22. Thiam AR, Farese RV Jr and Walther TC (2013) The biophysics and cell biology of lipid droplets. Nat Rev Mol Cell Biol 14, 775-786

23. Wilfling F, Haas JT, Walther TC and Farese RV Jr (2014) Lipid droplet biogenesis. Curr Opin Cell Biol 29, 39-45

24. Wilfling F, Haas JT, Walther TC and Farese RV Jr (2009) The endoplasmic reticulum enzyme DGAT2 is found in mitochondria-associated membranes and has a mitochondrial targeting signal that promotes its association with mitochondria. J Biol Chem 284, 5352-5361

25. Wilfling F, Wang $\mathrm{H}$, Haas JT et al (2013) Triacylglycerol synthesis enzymes mediate lipid droplet growth by relocalizing from the ER to lipid droplets. Dev Cell 24, 384-399

26. Cheng D, Meegalla RL, He B, Cromley DA, Billheimer JT and Young PR (2001) Human acyl-CoA:diacylglycerol acyltransferase is a tetrameric protein. Biochem J 359, 707-714

27. McFie PJ, Stone SL, Banman SL and Stone SJ (2010) Topological orientation of acyl-CoA:diacylglycerol acyltransferase-1 (DGAT1) and identification of a putative active site histidine and the role of the $\mathrm{n}$ terminus in dimer/tetramer formation. J Biol Chem 285, 37377-37387

28. Zhang J, Xu D, Nie J et al (2014) Monoacylglycerol acyltransferase-2 is a tetrameric enzyme that selectively heterodimerizes with diacylglycerol acyltransferase-1. J Biol Chem 289, 10909-10918

29. Jin Y, McFie PJ, Banman SL, Brandt C and Stone SJ (2014) Diacylglycerol acyltransferase-2 (DGAT2) and monoacylglycerol acyltransferase-2 (MGAT2) interact to promote triacylglycerol synthesis. J Biol Chem 289, 28237-28248

30. Hall AM, Soufi N, Chambers KT et al (2014) Abrogating monoacylglycerol acyltransferase activity in liver improves glucose tolerance and hepatic insulin signaling in obese mice. Diabetes 63, 2284-2296

31. Soufi N, Hall AM, Chen Z et al (2014) Inhibiting monoacylglycerol acyltransferase 1 ameliorates hepatic metabolic abnormalities but not inflammation and injury in mice. J Biol Chem 289, 30177-30188

32. Alam M, Gilham D, Vance DE and Lehner R (2006) Mutation of F417 but not of L418 or L420 in the lipid binding domain decreases the activity of triacylglycerol hydrolase. J Lipid Res 47, 375-383

33. Cao J, Zhou Y, Peng H et al (2011) Targeting Acyl-CoA:diacylglycerol acyltransferase 1 (DGAT1) with small molecule inhibitors for the treatment of metabolic diseases. J Biol Chem 286, 41838-41851 